Maria Cristina C. da Silva, MD'

Joel A. Romualdez, MD',

Norberto V. Martinez, MD ${ }^{1,2}$

'Department of Otolaryngology Head and Neck Surgery St. Luke's Medical Center

2 Department of Otolaryngology Head and Neck Surgery

Santo Tomas University Hospital
Correspondence: Maria Cristina C. da Silva, MD Department of Otolaryngology Head and Neck Surgery

St. Luke's Medical Center

279 E. Rodriquez Ave, Quezon City 1102

Philippines

Telefax: (632) 7275543

E-mail: peachiemd@yahoo.com

Reprints will not be available from the author.

No funding support was received for this study. The authors signed a disclosure that they have no proprietary or financial interest with any organization that may have a direct interest in the subject matter of this manuscript, or in any product used or cited in this report

Presented at the Interesting Case Contest (2nd Place) Philippine Society of Otolaryngology - Head and Neck Surgery Midyear Convention, Puerto Princesa City, Palawan, April 2005.

\section{Inflammatory Pseudotumor of an Orbito- Maxillary Mass Masquerading as a Malignancy}

\section{ABSTRACT}

Objective: To present a case of a benign orbito-maxillary tumor behaving as an invasive, expansive malignancy.

Design: Case report

Setting: A tertiary care hospital in Metro Manila

Patient: A $4 \frac{1}{2}$-year-old boy with a seven month history of right orbito-maxillary mass, proptosis and epiphora.

Result: A series of biopsies were done before a definite diagnosis was made due to inconsistencies in the histopathologic findings, clinical picture, and the radiologic presentation of the case. After diagnosis, appropriate intervention resulted in a dramatic decrease in the size of the mass. At present, the patient is disease-free and asymptomatic.

Conclusion: Histopathologic diagnosis of Inflammatory Pseudotumor is difficult and differentiating it from malignant tumors is often a concern for otolaryngologists and pathologists. In spite of an initial malignant biopsy result, the combination of clinical signs and symptoms and radiologic findings of an infiltrative mass lesion, should not discount the possibility of a benign entity such as Inflammatory Pseudotumor for which treatment is conservative.

Keywords: Orbito-Maxillary Mass, Inflammatory Pseudotumor, Pseudotumor, Orbital Pseudotumor

AN ORBITO-MAXILLARY mass may primarily be an orbital lesion extending into the maxillary sinus or a primary maxillary sinus lesion extending into the adjacent orbit. We present a case of $41 / 2$-year-old boy with an invasive right orbito-maxillary mass.

\section{CASE REPORT}

Seven months prior to consult, our patient presented with right lower eyelid swelling (Figure 1-A and 1-B) accompanied by non-productive cough and watery nasal discharge. There was no history of trauma. Maternal and birth history were unremarkable. Developmental history was at par with age. Past medical history included an allergy to contrast (dye) with a family history of brain cancer (maternal grandmother) and leukemia (paternal uncle). He was brought to an ear, nose and throat (ENT) specialist who diagnosed sinusitis and gave unrecalled antibiotics for 2 weeks which did not improve his condition.

Six months prior, the persistence of the symptoms prompted a consultation with another ENT specialist. Orbital magnetic resonance imaging (MRI) revealed a $3.2 \times 2.7 \times 2.8 \mathrm{~cm}$ mass within the inferior half of the right orbit and the right maxillary sinus with destruction of the orbital floor (Figure 2). There was also superior displacement and proptosis of the right globe.

He was referred to an ophthalmologist who biopsied the mass via an infraciliary approach. The report revealed "small round cell tumor to consider non-Hodgkin's lymphoma, Neuroendocrine tumor "(Figure 3) with a note from the pathologist that the specimen had 
excessive crushing artifact precluding a definite diagnosis. A repeat biopsy was suggested.

A trial of oral Prednisone at $0.2 \mathrm{mg} / \mathrm{kg} /$ day or $4 \mathrm{mg}$ daily for 1 month apparently decreased the size of the mass. The dose was then tapered to $2 \mathrm{mg}$ once a day then $1 \mathrm{mg}$ daily for 2 weeks each but the mass increased in size again. After 2 months of steroid therapy, an orbital computed tomography (CT) scan revealed a $3.6 \times 2.7 \times 2.7 \mathrm{~cm}$ mass within the right orbit and maxillary sinus (Figure 4) slightly larger than previously seen on MRI. Orbital floor lysis, as well as the superior displacement and proptosis of the right globe, were still evident.

The patient was referred to our institution for further management. He presented with a $2 \times 2 \mathrm{~cm}$ mass over the right lower eyelid and slight proptosis of the right eyeball with unremarkable visual acuity, extraocular muscle (EOM) motility, and fundoscopic exam. An incision biopsy via Caldwell-luc approach revealed "small round cell tumor, consider olfactory neuroblastoma" (Figure 5).

Special staining was negative for Chromogranin and LCA (leukocyte common antigen) but NSE (neuron-specific enolase) was positive, consistent with olfactory neuroblastoma. A positive NSE does not confirm the diagnosis nor does a negative chromogranin and LCA rule it out because it is not unusual for this kind of tumor to stain only focally. A repeat biopsy was again recommended by the pathologist. We requested a CT scan of the paranasal sinuses (Figure 6), which did not show interval changes from previous $\mathrm{CT}$ and MRI studies.

Closer examination of all radiologic studies revealed an unremarkable olfactory cleft with no sign of sinus wall bowing characteristic of an olfactory neuroblastoma. Clinically, the mass had not increased in size though 6 months had passed since the first biopsy. Doubting the diagnosis of olfactory neuroblastoma for lack of clinical and radiologic corroboration, another biopsy was done. The histopathologic reading of "chronic and acute inflammation negative for malignancy" (Figure 7) was not compatible with the bony lysis seen on CT and MRI.

We decided to debulk the tumor for both diagnostic and therapeutic purposes. The specimen was signed out as "Inflammatory Pseudotumor, right infraorbital area" (Figure 8).

The patient was given oral Prednisone at $2 \mathrm{mg} / \mathrm{kg} /$ day or $40 \mathrm{mg}$ daily for 2 weeks, $32 \mathrm{mg}$ once a day for 2 weeks, $24 \mathrm{mg}$ once a day for 2 weeks and $24 \mathrm{mg}$ daily for 4 weeks. 5 months post-surgery, the patient remained asymptomatic with no sign of recurrence of the mass (Figure 9). A repeat CT scan of the paranasal sinus revealed significant regression of the previously noted inferior orbital soft tissue mass ( $1 \mathrm{x}$ $0.4 \mathrm{~cm}$ ) (Figure 10-A). The proptosis and superior displacement of the right globe was no longer appreciated (Figure 10-B). Steroid therapy was discontinued and a 6-month review scheduled.

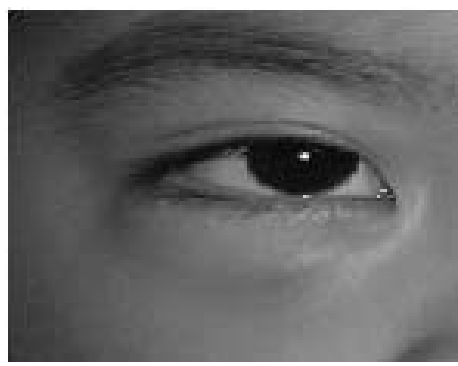

FIGURE 1-A: Right lower eyelid swelling

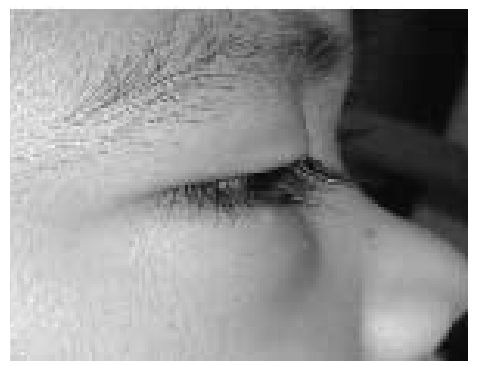

FIGURE 1-B: View from the right side

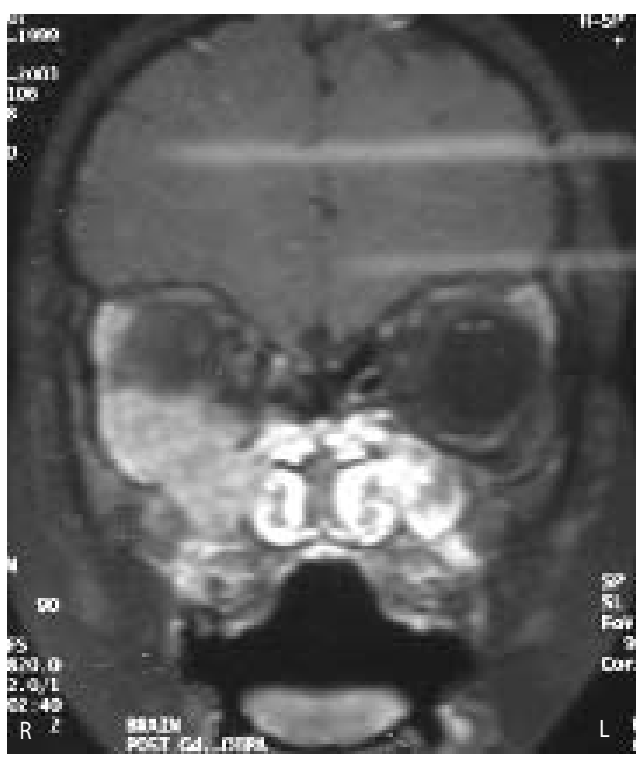

FIGURE 2: Orbital MRI: Mass within the inferior half of the right orbit and right maxillary sinus

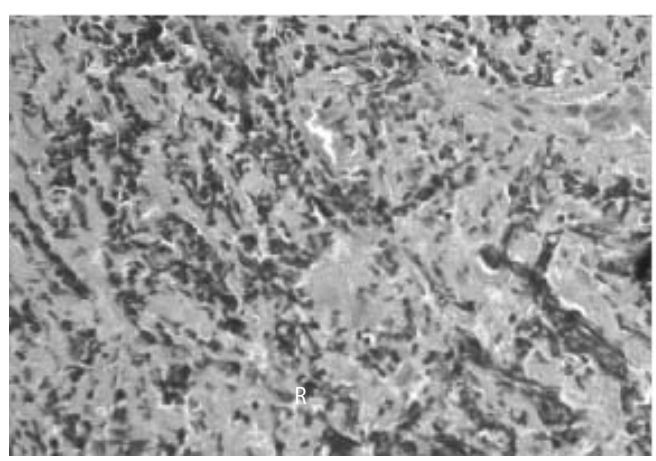

FIGURE 3: Small round cell tumor to consider NonHodgkin's Lymphoma, Neuroendocrine tumor 
CASE REPORTS

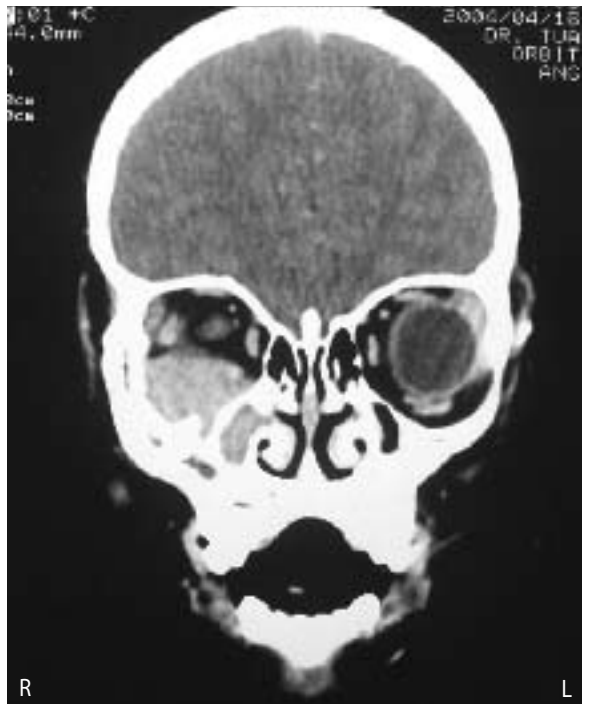

FIGURE 4: Orbital CT: Slightly larger mass within the right orbit

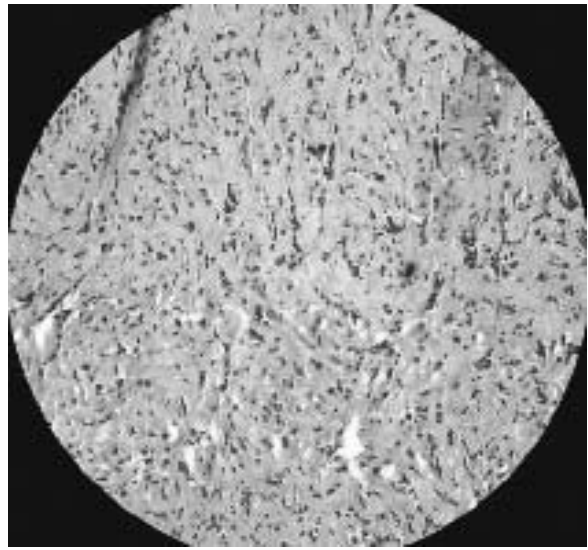

FIGURE 5: Small round cell tumor, consider olfactory neuroblastoma

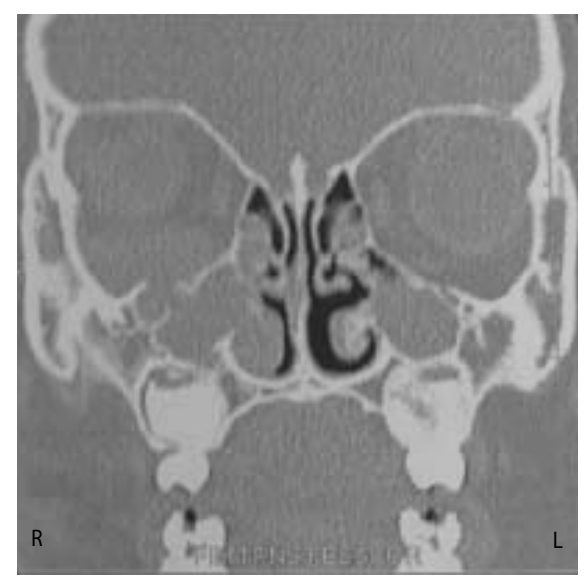

FIGURE 6: PNS CT: No significant interval changes

\section{DISCUSSION}

Orbital mass lesions may arise primarily within the orbit, extend from contiguous structures, or be metastatic from a distant primary malignancy. Primary orbital masses include congenital, vascular, neural, and mesenchymal tumors. Other types of lesions masquerading as true neoplasms include lymphoproliferative disorders, autoimmune diseases, and infectious processes. Congenital lesions include dermoid cysts, hamartomas and teratomas. The following table (Table 1) shows the most common pediatric tumors which present in the orbital area.

An olfactory neuroblastoma was considered in our second biopsy but $C T$ and MRI studies of our patient did not support this diagnosis. Olfactory neuroblastoma (Esthesioneuroblastoma) displays a variety of imaging characteristics and aggressiveness, characterized by bowing of the sinus walls, usually replacing the turbinates, septum, and sinus walls with extension into contiguous areas ${ }^{12}$. Not only did CT and MRI studies of our patient not show any of these findings, the unremarkable olfactory cleft cast more doubt on this diagnosis.

The acute and chronic inflammation seen on the third biopsy was possible following initial response to steroids but does not explain the lysis or bone destruction evident in the CT and MRI studies. The great discrepancy between malignancy and mere inflammation prompted tumor debulking to obtain a larger specimen and once and for all, put this diagnostic dilemma to rest, finally revealing inflammatory pseudotumor.

The first specimen had excessive crushing artifacts. Microscopically, small round cells which stained blue were identified. Small round inflammatory cells seen clumped or grouped together can make them seem neoplastic. Because small round cells are non-specific, special stains are needed (Table 2). Unfortunately, the excessive crushing of the specimen made this virtually impossible to accomplish.

The next biopsy again showed small round blue cells in clusters suggesting a neoplasm. Special staining negative for Chromogranin and LCA but positive for NSE supported a neuroendocrine tumor such as olfactory neuroblastoma but was not confirmatory. NSE is not a specific marker and needs chromogranin to stain positive for the diagnosis to be consistent with olfactory neuroblastoma. The pathologist could not commit to a diagnosis and suggested another biopsy, which gave a clue to the inflammatory nature of this disease. Microscopically, inflammatory cells such as lymphocytes, plasma cells and histiocytes were seen. However, the specimen was signed out only as acute and chronic inflammation because such cells are non-specific findings in any inflammatory process. But these cells also could very well be present at the surface of any malignant tumor. Tumor debulking provided a clearer pattern of cellular distribution with inflammatory cells present throughout the whole specimen. The pathological picture of dense fibrous tissue with aggregates of lymphocytes, plasma cells and histiocytes is the hallmark of inflammatory pseudotumor, but this basis is non-specific ${ }^{3}$. It is precisely the non-specific nature of the lesion which makes diagnosis difficult and limits the diagnostic yield of small biopsies. It was not that the specimens submitted were inadequate; the non-specific nature of the lesion could only be confirmed by getting the specimen in toto - a practice not commonly employed when dealing 


\section{CASE REPORTS}

Table 1. Common Orbital tumors in the pediatric age group

\begin{tabular}{l|l|l|l|l}
\hline DISEASE & $\begin{array}{l}\text { AGE OF } \\
\text { PRESENTATION }\end{array}$ & INCIDENCE & $\begin{array}{l}\text { SEX } \\
\text { PREDILECTION }\end{array}$ & ORBITAL SIGN/SYMPTOM \\
$\begin{array}{l}\text { RHABDOMYOSARCOMA } \\
\text { RETINOBLASTOMA }\end{array}$ & $2-5$ yrs. Old & $4.5 /$ million & Male predominance & Unilateral proptosis \\
$\begin{array}{l}\text { NEUROBLASTOMA } \\
\text { (METASTATIC) }\end{array}$ & $\begin{array}{l}\text { Before } 5 \text { yrs old } \\
1 \text { in } 18,000\end{array}$ & None & Leukokoria or proptosis \\
$\begin{array}{l}\text { OLFACTORY } \\
\text { NEUROBLASTOMA }\end{array}$ & $10 /$ million & Male predominance & Proptosis \\
$\begin{array}{l}\text { HISTIOCYTOSIS } \\
\text { (CHRONIC FORM) }\end{array}$ & $\begin{array}{l}11-20 \text { and } \\
\text { Older age group }\end{array}$ & Rare & No predilection & Proptosis \\
\hline
\end{tabular}

with a suspicious malignant-looking mass in an inaccessible area in the head and neck.

Inflammatory pseudotumor (IPT) is a rarely occurring lesion with no identifiable local or systemic causes. The lesions mimic expansive, invasive, malignant tumors both clinically and radiologically, hence the term "pseudotumor". IPT most commonly involves the lung and orbit. It has been reported in nearly every site in the body but is most common in the orbit and rare in the sinuses ${ }^{5}$. In our case, we believe that the mass was primarily an orbital lesion which extended into the maxillary sinus and not the other way around.

The behavior of IPT can be quite unpredictable ${ }^{3}$. Some resolve spontaneously and others respond to corticosteroids. In some cases, tapering steroid doses cause lesions to recur. For lesions not responding to steroids, subtotal excision with or without radiotherapy may be required ${ }^{3}$.

IPT is an idiopathic inflammatory lesion although various stimuli may cause it to develop such as unrecognized organisms, minor trauma, smoking, and chronic irritation by cocaine abuse ${ }^{1}$. The characteristic feature of IPT lies not in the inciting agent but in its response to a trigger agent. According to Williams et $\mathrm{al}^{4}$, the underlying mechanism in the development of IPT is localized derangement in the immune response after a particular initial insult. Other authors relate IPT to production of mediators of inflammation which stimulate proliferation of fibroblasts, extravasation of neutrophils and activation of procoagulant activity of the vascular endothelium. It also induces production of acute phase reactants, proteolysis and neurologic disturbances'.

The most frequent symptom is swelling and pain although other local symptoms depend on the site of involvement.

A very similar case was seen in Japan by Takashi Nakagawa et.al. ${ }^{2}$ where a 63 year old male presented with left eye lacrimation and exopthalmos. CT and MRI studies strongly suggested a malignant tumor of the maxillary sinus. A biopsy was also done which revealed IPT. Like our case, this patient did not respond to corticosteroids. Because total excision of the mass could not be achieved, debulking was done and there have been no observed further changes in the size of the mass several months after.

Another study done in New York by PM Som et al ${ }^{6}$ discussed 6 cases of IPT of the maxillary sinus. This study showed that IPT of the maxillary

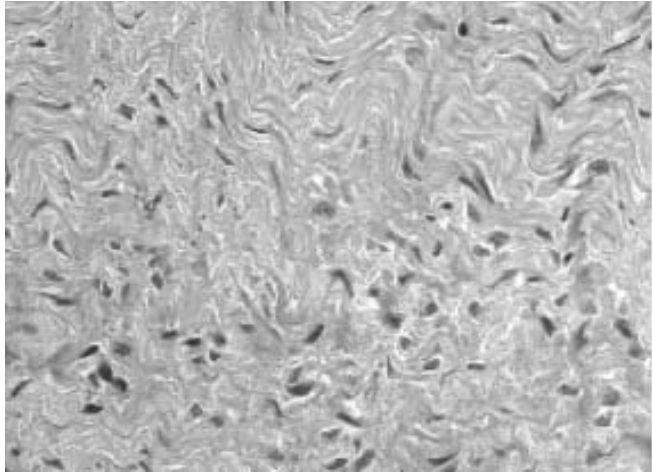

FIGURE 7: Chronic and acute inflammation negative for malignancy

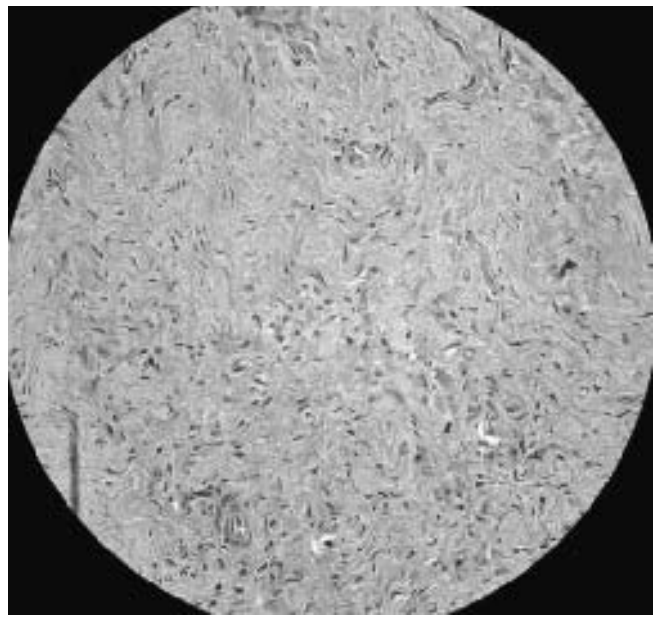

FIGURE 8: Inflammatory pseudotumor

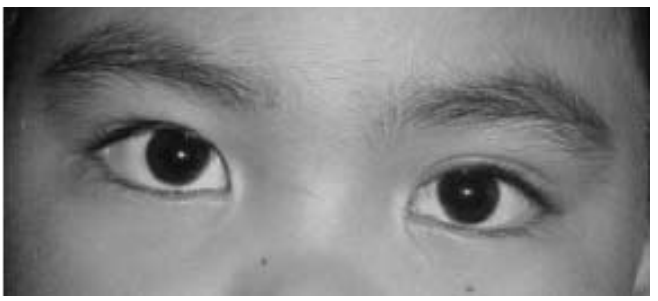

FIGURE 9: The patient was asymptomatic 6 months post-surgery 
CASE REPORTS

Table 2.

\begin{tabular}{l|l} 
IMMUNOHISTOCHEMICAL STAINS & TUMORS \\
LCA & Lymphoma \\
Cytokeratin & Epithelial tumors \\
NSE & Neuroendocrine tumors \\
Desmin & Muscle tumors \\
Vimentin & Mesenchymal tumors \\
Chromogranin & Neuroendocrine tumors \\
Synaptophysin & Neuroendocrine tumors \\
\hline
\end{tabular}

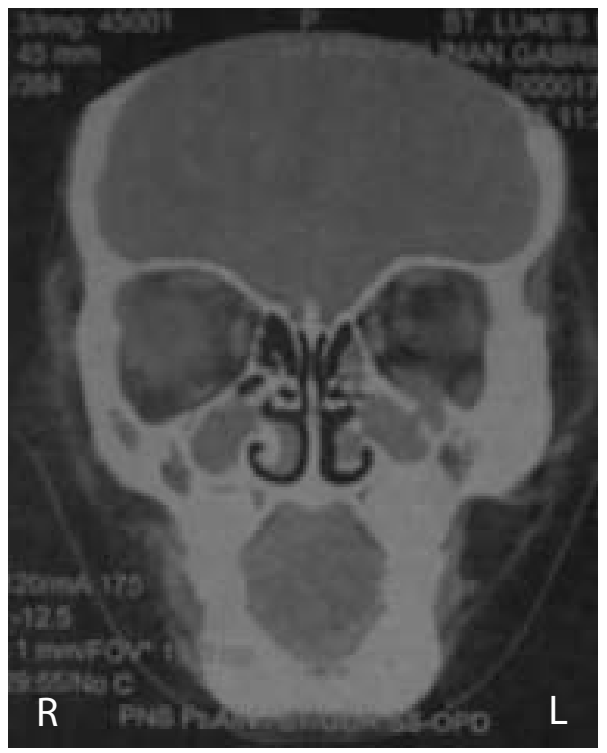

FIGURE 10-A: Regression of the globe soft tissue swelling

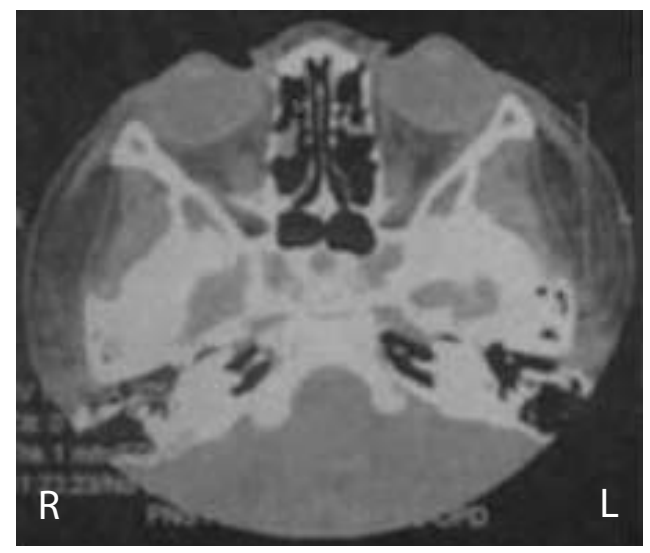

FIGURE 10-B: Proptosis of the right was no longer evident sinus causes bone changes on $\mathrm{CT}$ and $\mathrm{MRI}$, findings that mimic a malignant tumor.

Heathcote and Safneck ${ }^{3}$ reported a case of a 5-year-old boy who presented with eyelid swelling and was admitted with a diagnosis of orbital cellulitis. A CT scan performed the day after admission revealed a mass in the lateral orbit. Rather than the presumptive diagnosis of rhabdomyosarcoma, a biopsy revealed sclerosing IPT.

Among the different ancillary procedures, CT scan has proven to be of value in defining the extension of IPT and its response to treatment. In the orbit, the most frequent radiologic characteristics are retrobulbar fatty infiltration, proptosis, EOM enlargement and apical fat edema. In extraorbital locations in the head and neck, a mass lesion with sharp enhancement is consistently reported. IPT has an aggressive appearance and there is usually bone involvement like erosion, remodeling or sclerosis.

The unifying histologic feature of IPT is the highly variable mixture of bland-looking spindle cells and inflammatory cells ${ }^{1}$. The liberation of cytotoxic proteins from eosinophil granules may promote fibrosis and may also contribute to the degeneration of the extraocular muscles in IPT.

Histopathologic diagnosis of IPT is difficult and differential diagnosis from malignant tumors is often a concern for otolaryngologists and pathologists. Hence, coordination between these 2 specialties facilitates handling such difficult cases as this. The role of the ENT specialist in correlating history, physical examination, ancillary procedures and histopathology in spite of disparities cannot be overemphasized. In spite of an initial malignant biopsy result, the combination of clinical signs and symptoms and radiologic findings of an infiltrative mass lesion, should not discount the possibility of a benign entity such as IPT for which treatment is conservative, and unnecessary and potentially mutilating surgery can be avoided.

\section{ACKNOWLEDGEMENTS:}

We thank Dr. Anthony Calibo for help with statistical analysis, Dr. Francisco Narciso for technical assistance and Drs. Gretchen Navarro-Locsin and Bernabe Singson for their scientific advise.

\section{REFERENCES}

1. Sofie DV, Hermans R, Sciot R, Crevits I, Marchal G. Extraorbital inflammatory pseudotumor of the head and neck: CT and MR findings in three patients. Am J Neuroradiol. 1999;20(6):1133-39.

2. Nakagawa $\mathrm{T}$, Hatttori $\mathrm{K}$, Iwata $\mathrm{N}$, Hoshino T, Sasaki T. A case of inflammatory pseudotumor in the maxillary sinus mimicking malignancy. Practica Oto-Rhino-Laryngologica. 2002;95(4):29.

3. Heathcote J, Safneck J. Sclerosing inflammatory pseudotumor of orbit. Proceedings of the United States \& Canadian Academy of Pathology Annual Meeting; 2002 Feb 24; Chicago , Illinois

4. Williams SB. Inflammatory pseudotumor of the major salivary glands: clinicopathologic and immunohistochemical analysis of six cases. Otolaryngol Head Neck Surg. 1993;109(16):548-51.

5. Maldijan JA, Norton Kl, Som PM. Inflammatory pseudotumor of the maxillary sinus in a 15-year-old boy. Am J Neuroradiol. 1994;4(15):784-6.

6. Som PM, Brandwein MS, Maldijan C, Reino AJ, Lawson W. Inflammatory psuedotumor of the maxillary sinus: CT and MR findings in six cases. AJR. 1994;163(3):689-92.

7. Pickuth D, Obrunner K, Spielman RP. Computed tomography and magnetic resonance imaging features of olfactory neuroblastoma: an analysis of 22 cases. Clin Otolaryngol. 1999;24(5):457-61. 